OPEN ACCESS

Edited by:

Mohammad Anwar Hossain,

Bangladesh Agricultural University,

Bangladesh

Reviewed by:

Juniie Zhang,

Sichuan Agricultural University, China

Yu Zhao,

Huazhong Agricultural University,

China

*Correspondence:

Weiqun Liu

liuweiqun2004@126.com;

Hongxiang Guo

guohongxiang06@126.com

†These authors have contributed equally to this work.

Specialty section:

This article was submitted to Crop Science and Horticulture,

a section of the journal

Frontiers in Plant Science

Received: 15 February 2016

Accepted: 14 April 2016

Published: 28 April 2016

Citation:

LiF, Zhang H, Wang S, Xiao W, Ding C,

Liu W and Guo H (2016) Identification of Topping Responsive Proteins in Tobacco Roots.

Front. Plant Sci. 7:582.

doi: 10.3389/fpls.2016.00582

\section{Identification of Topping Responsive Proteins in Tobacco Roots}

\author{
Fei Li $^{1 \dagger}$, Huizhen Zhang ${ }^{2 \dagger}$, Shaoxin Wang ${ }^{1 \dagger}$, Wanfu Xiao ${ }^{1}$, Chao Ding ${ }^{1}$, Weiqun Liu ${ }^{1 *}$ and \\ Hongxiang Guo ${ }^{1 *}$
}

${ }^{1}$ College of Life Sciences, Henan Agricultural University, Zhengzhou, China, ${ }^{2}$ College of Public Health, Zhengzhou University, Zhengzhou, China

The process of topping elicits many responses in the tobacco plant, including an increase in nicotine biosynthesis, and the secondary growth of roots. Some topping responsive miRNAs and genes have been identified in our previous study, but the mechanism of the tobacco response to topping has not yet been fully elucidated. In this study, topping responsive proteins isolated from tobacco roots were screened using two-dimensional electrophoresis. Of the proteins identified, calreticulin and auxinresponsive protein indole acetic acid (IAA9) were involved in the secondary growth of roots; leucine-rich repeat disease resistance, heat shock protein 70 , and farnesyl pyrophosphate synthase 1 were involved in the wounding stress response; and F-box protein played an important role in promoting the ability of nicotine synthesis after topping. In addition, we identified five tobacco bHLH proteins (NtbHLH, NtMYC1a, NtMYC1b, NtMYC2a, and NtMYC2b) related to nicotine biosynthesis. NtMYC2 was suggested to be the main positive transcription factor, with NtbHLH protein being a negative regulator in the jasmonic acid (JA)-mediated activation of nicotine biosynthesis after topping. Tobacco topping activates a comprehensive range of biological processes involving the IAA and JA signaling pathways, and the identification of proteins involved in these processes will improve our understanding of the topping response.

Keywords: tobacco, topping, roots proteome, physiology response, secondary growth, nicotine synthesis

\section{INTRODUCTION}

Tobacco is an important economic crop, with the leaf representing the primary product. In order to improve leaf quality and production, the flowering head and young leaves of the tobacco plant are removed when the first flower of inflorescence appears. This cultivation technique for fluecured tobacco is known as topping. Topping can switch the plant from its reproductive phase to its vegetative phase by altering a number of biological processes in the plant, leading to changes in nicotine biosynthesis, the hormonal balance, root development, and the source-sink relationship (Baldwin et al., 1994; Li et al., 2007).

Nicotine, a secondary metabolite of the tobacco stress response, is an important raw material for pharmaceuticals, bio-pesticides, and biochemical reagents. Therefore, nicotine not only plays a key role in the plant's natural defense against herbivores and insects, but it is also a crucial substance

Abbreviations: bHLH, basic helix-loop-helix; CRT, calreticulin; FPPS, farnesyl pyrophosphate synthase 1; GH3, gretchen hagen 3; IAA, indole acetic acid; IEF, isoelectric focusing; JA, jasmonic acid; MeJA, methyl jasmonate; 2,4-D, 2,4dichlorophenoxyacetic acid; ODC, ornithine decarboxylase; PMT, putrescine $N$-methyltransferase; SAMS, $S$-adenosyl-Lmethionine synthetase; SAUR, small auxin up RNA; QPT, quinolinic acid phosphoribosyl transferase. 
contributing to the commercial value of the tobacco leaf. Nicotine is synthesized predominantly in tobacco roots, and then transported into leaves. Nicotine biosynthesis can be influenced by many factors, including developmental stages, hormones, and both biotic and abiotic stressors. The up-regulation of nicotine synthesis is a typical response of tobacco roots to topping. PMT, ODC, SAMS, and QPT are some key enzymes in nicotine synthesis. The activities of these enzymes are known to increase following tobacco topping, suggesting that topping can enhance the ability of roots to synthesize nicotine (Saunders and Bush, 1979). The expression of PMT, ODC, SAMS, and QPT increased in cell cultures of Nicotiana tabacum treated with $\mathrm{MeJA}$, indicating that MeJA has a regulatory role in activating nicotine synthesis (Imanishi et al., 1998; Shoji et al., 2000; Winz and Baldwin, 2001). JA is a wounding responsive signaling molecule. JA levels increased 10-fold in damaged leaves within $90 \mathrm{~min}$, and an increase was observed in the roots at $2 \mathrm{~h}$ after wounding (Baldwin et al., 1997).

Since tobacco topping is a form of mechanical wounding, it can induce JA synthesis in the wounded area. In addition, removal of the apical meristem reduces the source of IAA in tobacco aboveground parts after topping, which changes the distribution of IAA in tobacco plant. JA and IAA have been identified as two key phytohormones in the response of tobacco to topping (Zhang and Baldwin, 1997; Shoji et al., 2000; Shi et al., 2006). Furthermore, two bHLH transcriptional factors (NbbHLH1 and NbbHLH2) have been identified as positive regulators, and a third factor (NbbHLH3) as a negative regulator in the JA-mediated activation of nicotine synthesis (Todd et al., 2010). Therefore, multiple intersecting signal transduction pathways and diverse transcriptional regulatory factors are involved in the upregulation of nicotine synthesis after topping ( $\mathrm{Xu}$ and Timko, 2004). Further work is required, however, to fully elucidate the mechanisms involved in the regulation of nicotine synthesis by JA and IAA.

The secondary growth of roots is another important response to topping in the tobacco plant. It has been reported that the biomass, number, and activity of the roots increased significantly after topping (Zhang et al., 2006). Topping decreased the content of IAA in tobacco aboveground parts, which altered the source-sink relationship such that tobacco roots become an important growth center. However, the mechanism of secondary growth of tobacco roots induced by topping remains unclear.

Some previous studies have evaluated tobacco topping responsive genes. A set of 60 cDNAs was screened using subtractive hybridization of a phage library before and after topping. The differentially expressed genes identified were related to signal transduction pathways, transcription, translation, wounding stress, and alkaloid biosynthesis, including arginine decarboxylase (ADC), ODC, and SAMS (Wang et al., 2000). Furthermore, virus-induced gene silencing led to the identification of six transcription factors involved in the regulation of nicotine synthesis by JA: NbERF1, NbbHLH1, NbbHLH2, NbbHLH3, NbARF1, and NbHB1 (Todd et al., 2010). Genes and miRNAs showing differential expression levels before and after topping were analyzed by combining miRNA deep sequencing and suppression subtractive hybridization. This led to the proposition of a miRNA-mediated model for the topping response in flue-cured tobacco (Qi et al., 2012). Further, these authors demonstrated that NtNAC, a target transcription factor of miR164a, was involved in the auxin signaling pathway, and nicotine synthesis. Many studies investigating the tobacco topping response at a transcriptional level have thus been reported, the results of which make an important contribution to our understanding of the response of flue-cured tobacco to topping.

Topping results in a large and rapid change of JA and IAA content in the tobacco shoot, and a delayed response in the roots. Many molecular events at the protein level will certainly be successively involved in the response to topping. However, thus far, no studies have investigated the dynamic changes in the tobacco root proteome after topping. This study set out to screen topping responsive proteins in order to shed further light on the mechanisms involved in the response of tobacco plants to topping.

\section{MATERIALS AND METHODS}

\section{Plant Materials and Stress Treatments}

Tobacco (N. tabacum K326) plants in the field were topped when the first flower of inflorescence came into bloom. Tobacco plants allowed to grow without topping were used as a control. The roots of the tobacco plants were removed from the soil 10 days after topping. The number of lateral roots was compared between topped and control tobacco plants. The roots in the field were also sampled at $0,2,6$, and $24 \mathrm{~h}$ post-topping for proteomic analysis. These samples were immediately frozen in liquid nitrogen and then stored at $-80^{\circ} \mathrm{C}$ until analyzed.

Tobacco plants in pots were randomly divided into groups to undergo seven different stress treatments. All stress treatments were performed at the point when the first flower of inflorescence bloomed. Tobacco plants were not topped in any stress treatments other than the topping stress treatment. (1) Topping treatment: the flowering head and young leaves were removed; roots were sampled at $24 \mathrm{~h}$ after topping. (2) 2,4-D treatment: leaves were sprayed with $50 \mu \mathrm{M} 2,4-\mathrm{D}$ solution; roots were sampled at $24 \mathrm{~h}$ after spraying. (3) MeJA treatment: leaves were sprayed with $100 \mu \mathrm{M}$ MeJA solution; roots were sampled at $24 \mathrm{~h}$ after spraying. (4) Leaf damage treatment: leaves were punctured; roots were sampled at $24 \mathrm{~h}$ after treatment. (5) Cold treatment: plants were kept at $4^{\circ} \mathrm{C}$ for $48 \mathrm{~h}$; roots were sampled at the end of the $48 \mathrm{~h}$ treatment period. (6) Drought treatment: plants were kept without water for 20 days; roots were sampled at the end of the 20-day treatment period. (7) Salt treatment: plants were treated with $200 \mathrm{mM} \mathrm{NaCl}$ solution for 15 days; roots were sampled at the end of the 15 -day treatment period. All samples were immediately frozen in liquid nitrogen and then stored at $-80^{\circ} \mathrm{C}$ until used for analysis. 


\section{Extraction and Two-Dimensional Electrophoresis of Root Proteins}

Tobacco roots $(1 \mathrm{G})$ were ground into a fine powder in liquid nitrogen. Proteins were extracted with the trichloroacetic acidacetone-phenol method (Guo et al., 2015). After $1000 \mu \mathrm{g}$ of proteins were loaded into the immobilized strip $(24 \mathrm{~cm}$, pH 310), IEF was performed at $50 \mathrm{~V}$ for $12 \mathrm{~h}, 200 \mathrm{~V}$ for $3 \mathrm{~h}, 500 \mathrm{~V}$ for $1.5 \mathrm{~h}, 1,000 \mathrm{~V}$ for $0.5 \mathrm{~h}$, and $10,000 \mathrm{~V}$ for $3 \mathrm{~h}$ with a Protean IEF Cell system (Bio-Rad). The strips were then equilibrated for 15 min with buffer I (50 mM Tris- $\mathrm{HCl} \mathrm{pH} \mathrm{8.8,} 6 \mathrm{M}$ urea, $4 \%$ SDS, $20 \%$ glycerol, and $0.1 \%$ dithiothreitol) and $15 \mathrm{~min}$ with buffer II (50 mM Tris- $\mathrm{HCl} \mathrm{pH}$ 8.8, $6 \mathrm{M}$ urea, 4\% SDS, $20 \%$ glycerol, and $0.25 \%$ iodoacetamide). Protean Plus Dodeca Cell (Bio-Rad) was used to perform the second dimension of electrophoresis on $12.5 \%$ sodium dodecyl sulfate polyacrylamide gel. The electrophoresis was run at $50 \mathrm{~V}$ and $25^{\circ} \mathrm{C}$ until the samples deviated from the strips and then at $200 \mathrm{~V}$ until the bromophenol blue dye front reached the bottom of the gel. After staining with Coomassie brilliant blue R-250, the gels were scanned with ImageScanner II (Amersham Pharmacia) to obtain 2-DE image. The image was analyzed with PDQest8.0 software $^{1}$. Each spot was quantified as the accumulated intensity of the optical density in proportion to spot volume. Each protein sample was obtained from three independent tobacco plants, and the 2-DE analysis was performed in triplicate for each sample. Plants given no topping treatment were used as a control, and

${ }^{1}$ www.bio-rad.com/products/pdquest/ data were presented as fold change (increase or decrease) versus matched controls. The protein spots with a significant difference $(p<0.05)$ were considered to be "significantly differential proteins." The significantly differential proteins were identified by 4800 Plus MALDI TOF/TOF ${ }^{\mathrm{TM}}$ Analyzer (ABI). The default parameters of Mascot software (Matrix science Company) were used to perform sequence similarity searches against NCBInr Database [Viridiplantae (1093002) and EST_Nicotiana (412325)].

\section{Quantitative RT-PCR Analysis}

Total RNA was isolated from tobacco roots using TRIzol reagent (Invitrogen, Carlsbad, CA, USA). The reverse transcription reactions were performed with One Step PrimeScript RT-PCR Kit (Perfect Real Time). TaKaRa SYBR Premix Ex Taq ${ }^{\mathrm{TM}}$ II (Perfect Real Time) was used for quantitative real time PCR on a Bio-Rad IQ5 Real-Time PCR Detection System. The volume of the qRT-PCR reaction was $25 \mu \mathrm{L}$, and actin was used as the endogenous reference gene. Each reaction consisted of $2 \mu \mathrm{L}$ of product from the diluted reverse transcription reaction, $12.5 \mu \mathrm{L}$ of $2 \mathrm{x}$ SYBR Premix Ex Taq ${ }^{\mathrm{TM}}$ II, $0.5 \mu \mathrm{L}$ of primers (forward and reverse), and $9.5 \mu \mathrm{L}$ of nuclease-free water. The reactions were incubated at $95^{\circ} \mathrm{C}$ for $30 \mathrm{~s}$, followed by 40 cycles of $95^{\circ} \mathrm{C}$ for $5 \mathrm{~s}, 57^{\circ} \mathrm{C}$ for $30 \mathrm{~s}$, and $72^{\circ} \mathrm{C}$ for $30 \mathrm{~s}$. Three replicates were performed for each sample. Fold change was determined using the $2^{-\Delta \Delta C t}$ method and error bars represent the standard deviation (SD) of the mean. AVEDEV and Student's $t$-tests were used to determine SD of the mean

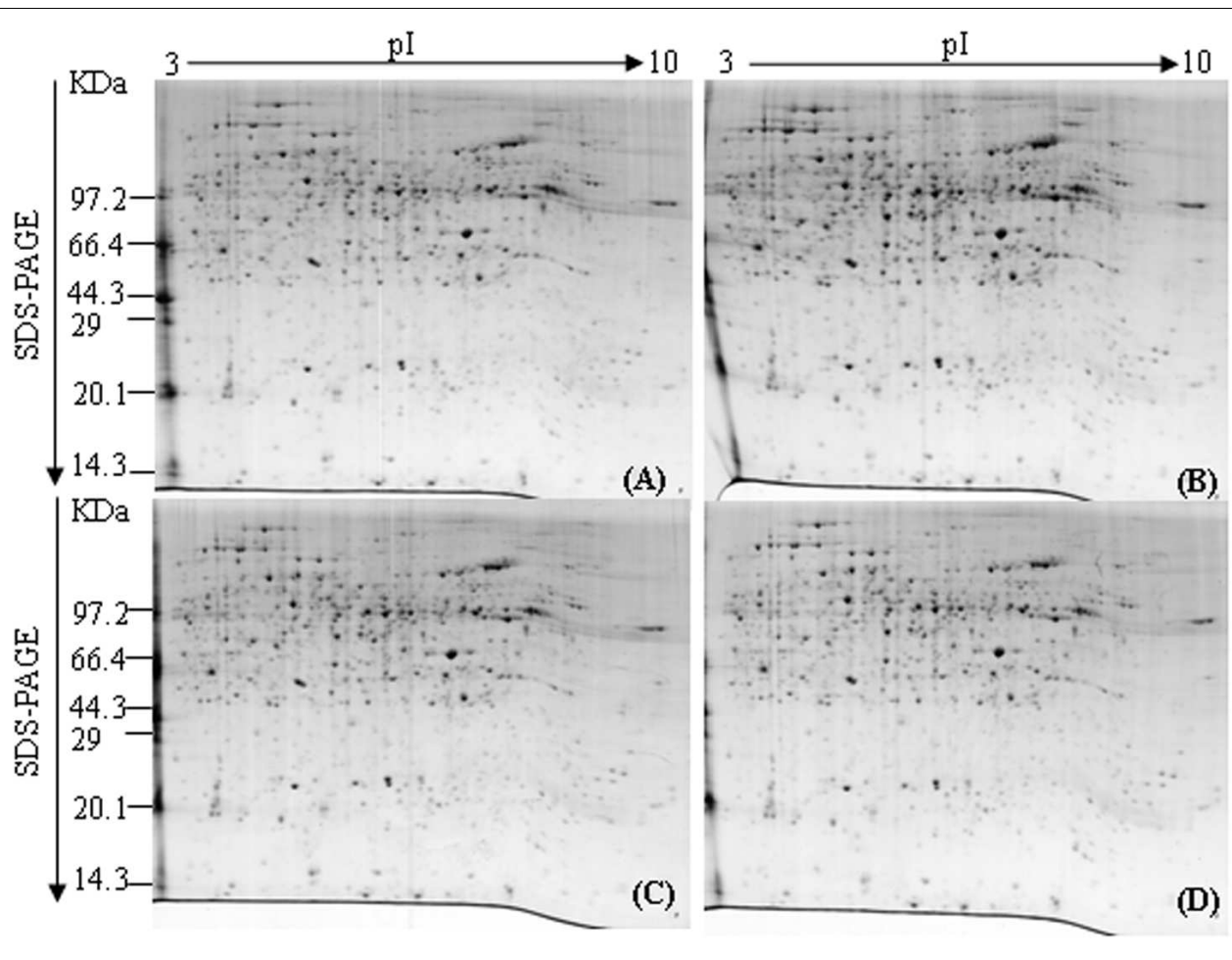

FIGURE 1 | 2DE of tobacco roots proteome before and after topping. (A) No topping; (B) $2 \mathrm{~h}$ after topping; (C) $6 \mathrm{~h}$ after topping; (D) $24 \mathrm{~h}$ after topping. $1000 \mu \mathrm{g}$ of proteins were loaded, and the gels were stained with Coomassie brilliant blue R-250. 
and significant differences between groups. The primer pairs used for quantitative RT-PCR are shown in Supplementary Table S1.

\section{RESULTS}

\section{Comparative Analysis of 2-DE Maps of Tobacco Root Proteome Following Topping}

To investigate the dynamic changes of tobacco roots proteome in response to topping, 2-DE analysis of the total proteins in roots before, and after topping was carried out. The 2DE electrophoretic maps obtained from tobacco roots are shown in Figure 1. The protein spots showed a broad distribution in the pI range from 3.0 to 10.0 and the mass range from 10 to $120 \mathrm{kDa}$. In all, 184 significantly differential protein spots were detected between before and after topping (Figure 2).

\section{Identification of Differential Proteins in Tobacco Roots Following Topping}

Thirteen significantly differential proteins were analyzed by MALDI-TOF-MS (Shown in Figure 3), and identified by searching the UniProt protein database (Table 1). These proteins included F-box protein, Leucine-rich repeat (LRR) disease resistance, CRT, Ras-related protein, bHLH, Heat shock protein $70 \mathrm{kDa}$ (HSP70), Auxin-responsive protein IAA9, V-type proton ATPase subunit B, FPPS and proteinase inhibitor.

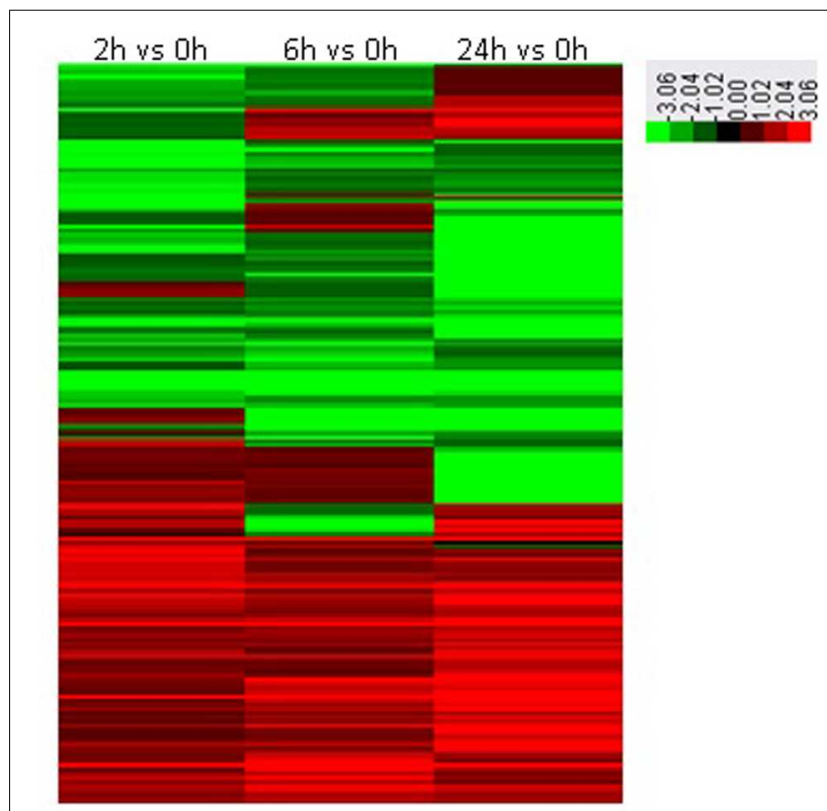

FIGURE 2 | Heat maps of significantly differential proteins following topping. 0 h: No topping; 2 h: 2 h after topping; 6 h: 6 h after topping; 24 h: $24 \mathrm{~h}$ after topping. No topping treatment was used as the control.

\section{Differential Proteins Related to Root Development Following Topping}

Plant root systems display considerable plasticity in response to endogenous and environmental signals. It has been previously shown that a change in root development is an important response of flue-cured tobacco to topping (Zhang et al., 2006). We found that the numbers of tobacco lateral roots markedly increased at 10 days after topping (Figure 4), indicating that growth of tobacco roots can be promoted by topping.

Calreticulin, an ER-localized $\mathrm{Ca}^{2+}$-binding protein, is a protein expressed differentially in the presence or absence of topping treatment. CRT plays a critical role not only in $\mathrm{Ca}^{2+}$ homeostasis but also in $\mathrm{Ca}^{2+}$ signaling mechanisms related to regeneration (Jin et al., 2005). Furthermore, it has been shown to modulate an array of responses to environmental stresses, such as cold and drought (Kim et al., 2013). As shown in Figure 5, the mRNA expression of CRT was up-regulated at $2 \mathrm{~h}$ after topping. This is consistent with the results of 2-DE, suggesting that CRT might play an important role in the growth of tobacco roots after topping.

The result of 2-DE demonstrated that IAA9 was downregulated after topping (Table 1). Aux/IAA genes are involved in auxin-related root development processes, such as primary root elongation and lateral root formation (Fukaki et al., 2002; Tatematsu et al., 2004; Uehara et al., 2008). As shown in Figure 5, the mRNA expression of IAA9 was down-regulated after topping, suggesting that IAA9 plays an important role in the formation of lateral roots after topping.

\section{Differential Proteins Related to Damage Response Following Topping}

Topping is a form of mechanical wounding. Plants have many molecules mediating a physiological response to wounding, such as JA and secondary metabolites. FPPS catalyzes the formation of farnesyl pyrophosphate (FPP) which is a key cellular intermediate in isoprenoid metabolic pathways. FPP is a biosynthetic precursor of steroid hormones, dolichols, and sesquiterpene phytoalexin. In addition, it has been reported that FPP has a role in signal transduction (Reilly et al., 2002). The proteomic analysis of tobacco roots showed that FPPS was markedly up-regulated after topping. As shown in Figure 6, the mRNA expression of FPPS was also up-regulated at $24 \mathrm{~h}$ after topping, suggesting that FPPS is involved in the response to topping damage. Hsp70 and LRR were the other two differential proteins. HSP70 is a stress responsive protein, and it was found to be up-regulated at the mRNA level after topping (Figure 6). In plants, the LRR disease resistance protein is the main class of disease resistance genes. The mRNA expression of LRR1 was markedly up-regulated at $24 \mathrm{~h}$ after topping, whereas the mRNA expression of LRR2 showed no obvious changes (Figure 6). Therefore, HSP70 and LRR1 are also implicated in the damage response following topping. 

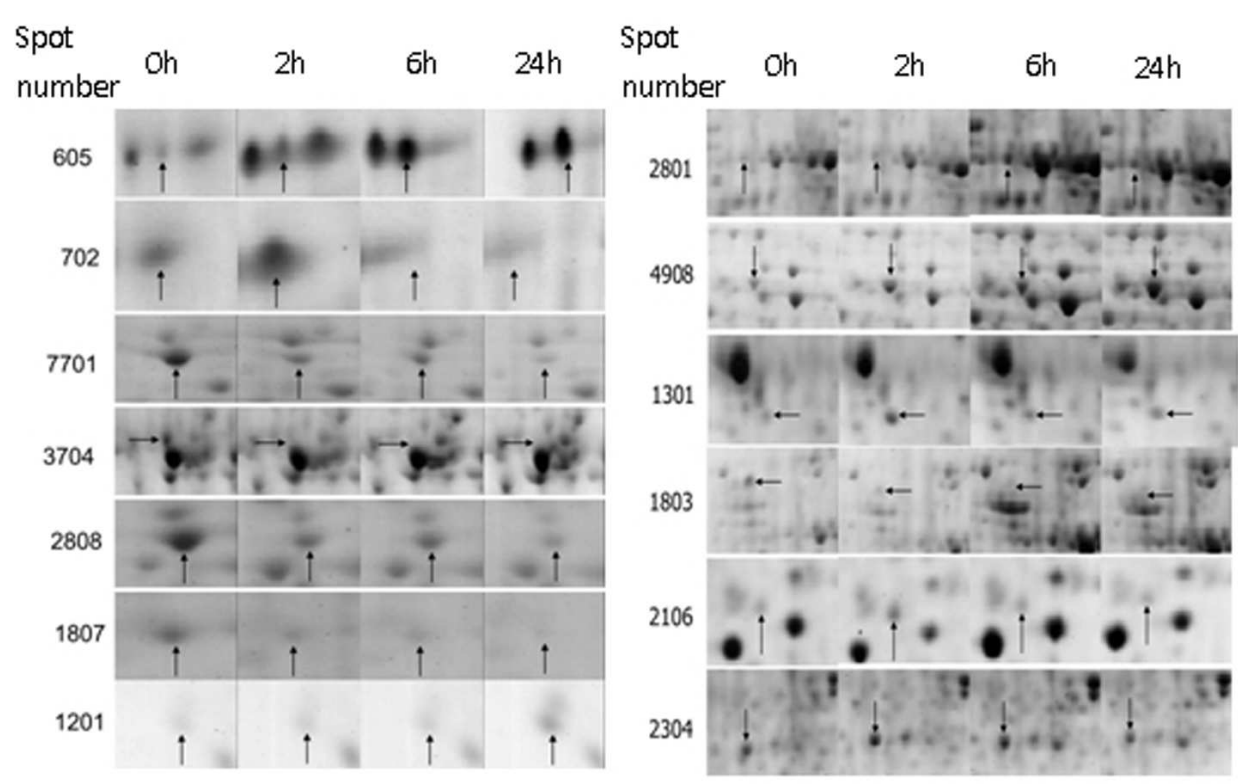

FIGURE 3 | The amplification map of differential proteins identified by MALDI-TOF-MS. $0 \mathrm{~h}:$ No topping; $2 \mathrm{~h}: 2 \mathrm{~h}$ after topping; $6 \mathrm{~h}: 6 \mathrm{~h}$ after topping; $24 \mathrm{~h}$ : $24 \mathrm{~h}$ after topping. No topping treatment was used as the control. Spot number: the numerical order of differential proteins.

TABLE 1 | The identification of differential proteins in tobacco roots following topping.

\begin{tabular}{|c|c|c|c|c|c|c|c|}
\hline Spot number & Protein name & UniProt accession number & MW & pl & \multicolumn{3}{|c|}{ Fold change } \\
\hline 1807 & Proteinase inhibitor & P01078 & 6.03 & 7.68 & -1.03 & -1.52 & -8.33 \\
\hline 7701 & LRR disease resistance & P84735 & 8.70 & 9.63 & -3.85 & -2.63 & -5.88 \\
\hline 1201 & F-box protein & Q6NPQ1 & 43.65 & 9.59 & +1.16 & +1.51 & +3.05 \\
\hline 702 & Tobacco calreticulin & Q40401 & 47.68 & 4.45 & +2.15 & -1.16 & -1.27 \\
\hline 1301 & Ras-related protein & P28187 & 24.14 & 4.98 & +2.67 & +1.87 & +1.94 \\
\hline 1803 & $\mathrm{bHLH}$ & A4D998 & 25.36 & 6.45 & -1.15 & -4.76 & -3.85 \\
\hline 2106 & Auxin-responsive protein IAA9 & AJ417876 & 28.43 & 7.57 & +2.13 & -1.28 & -1.22 \\
\hline 2304 & Heat shock protein $70 \mathrm{kDa}$ & P11143 & 70.87 & 5.22 & +2.28 & +2.35 & +1.22 \\
\hline
\end{tabular}

\section{Differential Proteins Related to the Regulation of Nicotine Biosynthesis Following Topping}

The increase in nicotine biosynthesis is an important response of tobacco to topping. Tobacco F-box protein and basic helix-loop-helix protein (NtbHLH and nta000375) were two proteins expressed differentially before and after topping (Table 1). The protein family is the key component of ubiquitin ligase E3 in the SCF complex and is related to many biological processes, including hormone signal transduction, plant growth and development, and abiotic and biotic stresses. $\mathrm{SCF}^{\mathrm{COI}}$ can bind JA-Ile, thus initiating the degradation of jasmonate ZIM-domain protein (JAZ), which is a negative regulator of JA responses (Wang and $\mathrm{Wu}, 2013$ ). The bHLH is a member of an important plant transcription factor family in response to environmental stresses. It has been reported that F-box protein and bHLH family proteins are involved in JA-mediated nicotine biosynthesis (Wang and $\mathrm{Wu}$, 2013). NbbHLH1 and NbbHLH2 were positive regulators, whereas NbbHLH3 had negative regulatory function in the JA-inducible nicotine synthesis (Todd et al., 2010). Therefore, it is unsurprising that the mRNA expression of F-box 2 and F-box3 proteins increased, and that the mRNA expression of F-boxl and NtbHLH (nta000375) protein decreased after topping (Figure 7). 


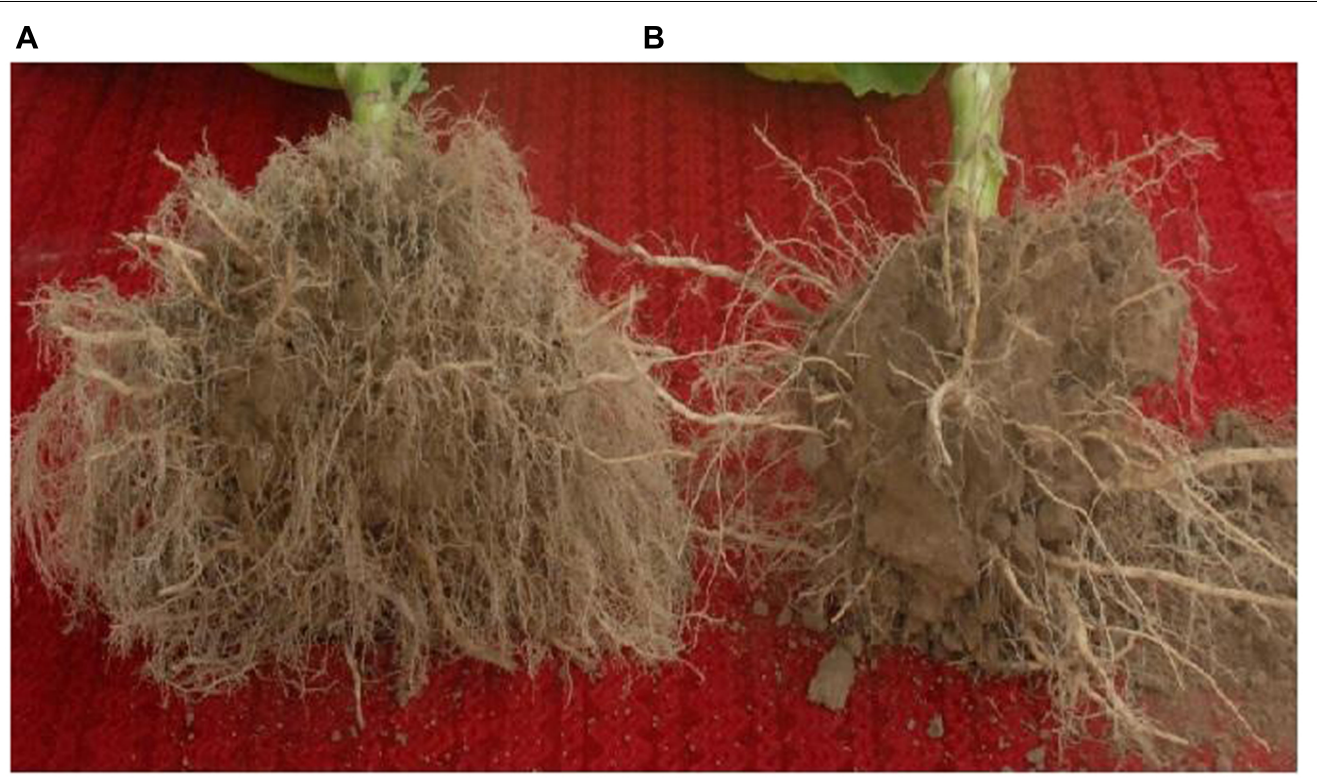

FIGURE 4 | The effect of topping on tobacco root development. The roots of tobacco plants were taken out of the soil and photographed at 10 days after topping. (A) tobacco plant with topping; (B) tobacco plant without topping.
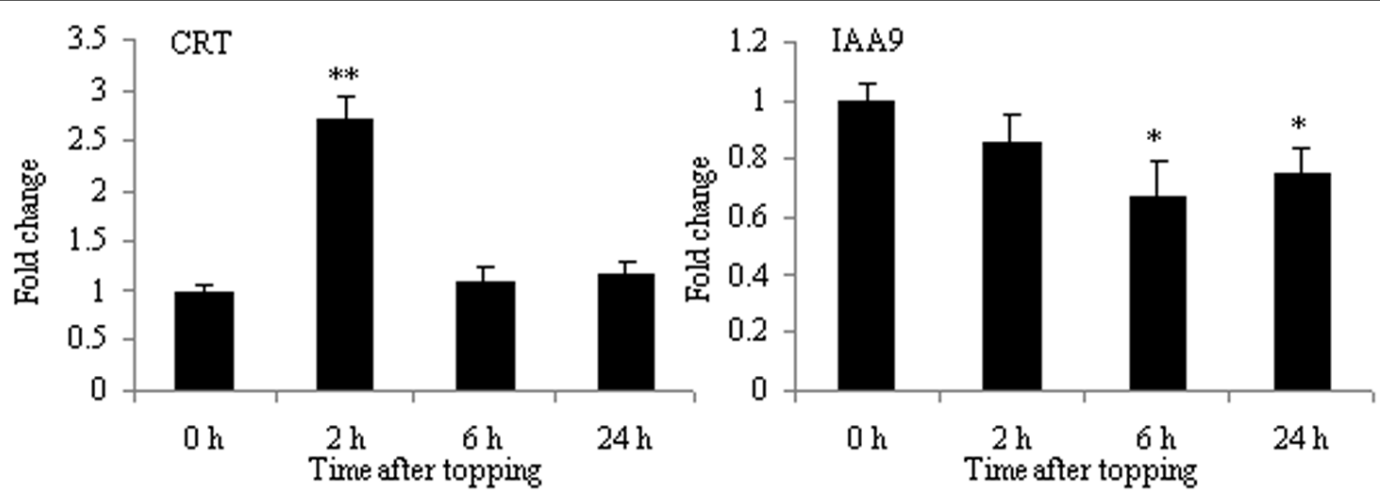

FIGURE 5 | RT-qPCR analyses of CRT and IAA9 in roots after tobacco topping. CRT, calreticulin; IAA9, auxin-responsive protein IAA9. Fold change was determined using the $2^{-\Delta \Delta C t}$ method and error bars represent the standard deviation (SD) of the mean. Statistical significance: ${ }^{*} p<0.05$ and ${ }^{* *} p<0.01$.

\section{Several bHLH Proteins Related to Nicotine Biosynthesis}

The bHLH family of proteins is a diverse group of functional transcription factors. There are $48 \mathrm{bHLH}$ proteins from TOBFAC databases in the tobacco plant ${ }^{2}$. A phylogenetic tree of tobacco bHLH family proteins was constructed (Figure 8). NtMYC1a/1b and NtMYC2a/2b belong to MYC subfamily, and it has been shown that NtMYC2a and $2 \mathrm{~b}$ are involved in regulating nicotine biosynthesis (Wang and $\mathrm{Wu}, 2013$ ). NtbHLH (nta000375) protein does not belong to MYC subfamily, so it has a different role in regulating nicotine biosynthesis than do MYC subfamily proteins.

Nicotine biosynthesis can be regulated by multiple biotic and abiotic stresses, such as drought stress, and herbivore or insect

${ }^{2}$ http://compsysbio.achs.virginia.edu/tobfac/ damage. PMT is the key enzyme in the nicotine biosynthetic pathway, and it regulates nicotine levels in tobacco. As shown in Figure 9, the mRNA expression of PMT can be increased by MeJA, 2,4-D, salt stress, cold stress, drought stress, leaf damage, and topping. NtbHLH protein was down-regulated by topping, leaf damage, and MeJA (Figure 10), indicating that $\mathrm{NtbHLH}$ protein is a negative regulator in the JA-mediated activation of nicotine synthesis. In addition, NtbHLH protein was up-regulated by $2,4-\mathrm{D}$, salt stress, cold stress, and drought stress, suggesting that it also has other functions in response to abiotic stress. The mRNA expression of NtMYC2a and NtMYC2b increased after MeJA, 2,4-D, leaf damage, and topping treatment, whereas NtMYC1a and NtMYC1b were markedly upregulated by MeJA, 2,4-D, and drought treatment. Hence, there are different responses to biotic and abiotic stresses between NtMYC1a/1b and NtMYC2a/2b. 

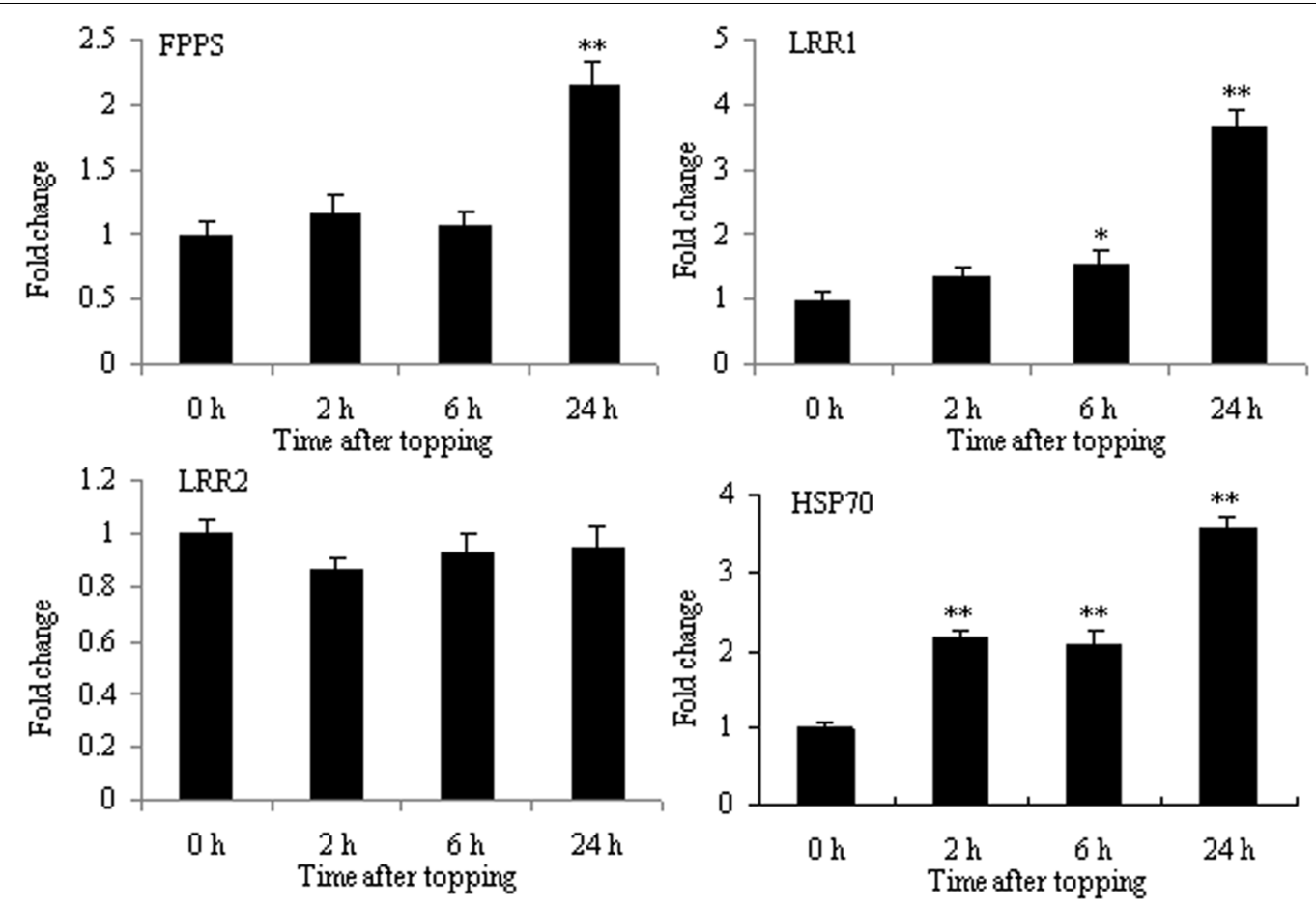

FIGURE 6 | RT-qPCR analyses of FPPS, HSP70, LRR1, and LRR2 in roots after tobacco topping. FPPS, farnesyl pyrophosphate synthase; HSP70, heat

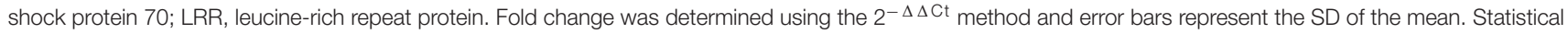
significance: ${ }^{*} p<0.05$ and ${ }^{* *} p<0.01$.
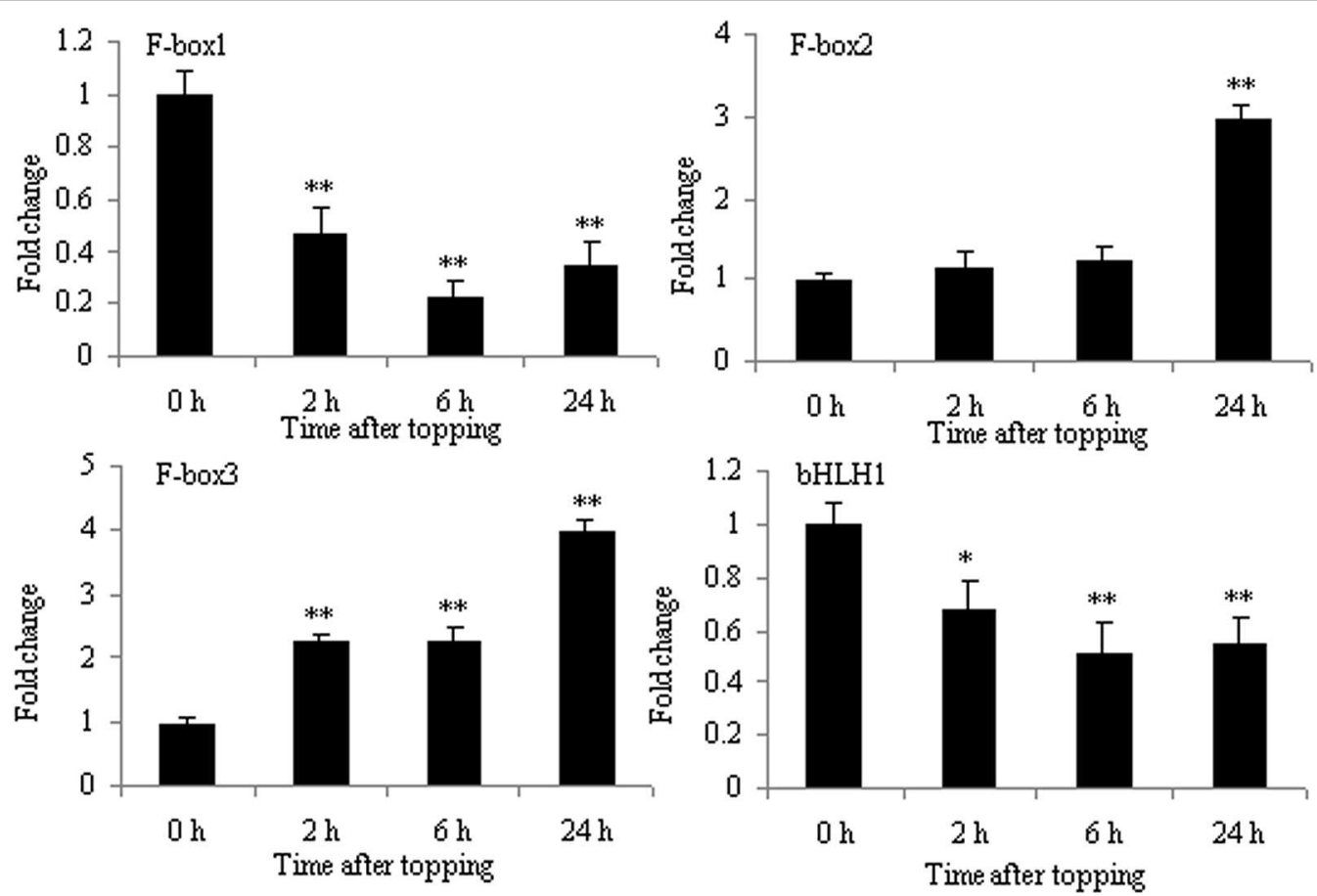

FIGURE 7 | RT-qPCR analysis of F-box1, F-box2, F-box3, and bHLH in roots after tobacco topping. bHLH, basic helix-loop-helix. Fold change was determined using the $2^{-\Delta \Delta \mathrm{Ct}}$ method and error bars represent the SD of the mean. Statistical significance: ${ }^{*} p<0.05$ and ${ }^{* *} p<0.01$. 


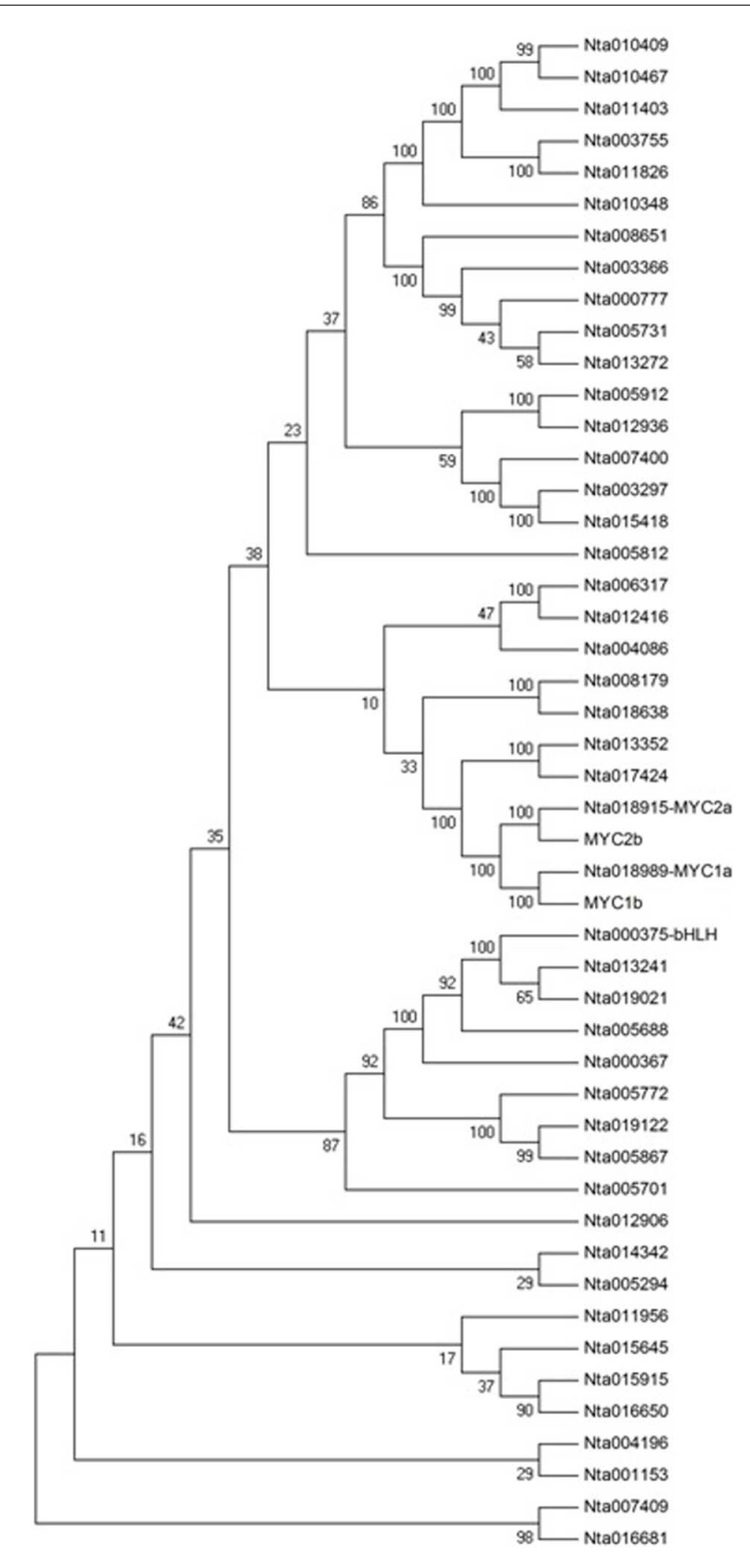

FIGURE 8 | Phylogenetic tree analysis of the bHLH transcription factor family in Nicotiana tabacum. MEGA4.0 software and default parameters were used in the phylogenetic tree analysis. The $48 \mathrm{bHLH}$ proteins are from TOBFAC databases in tobacco (http://compsysbio.achs.virginia.edu/tobfac/).

\section{DISCUSSION}

\section{Transformation of Growth Centre Promotes Secondary Growth of Roots after Topping}

Topping is the removal of flowering head and young leaves (apical meristem) which is a primary source of auxin in the

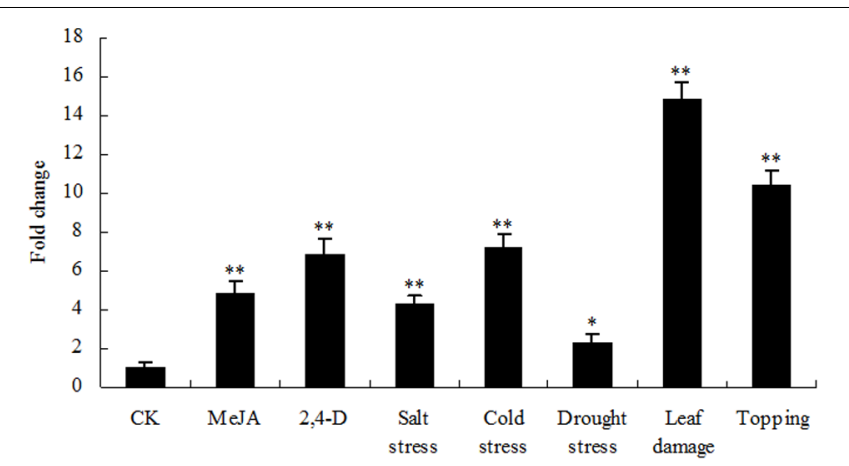

FIGURE 9 | RT-qPCR analysis of PMT in tobacco roots after stress treatment. Fold change was determined using the $2^{-\Delta \Delta C t}$ method and error bars represent the SD of the mean. Statistical significance: ${ }^{*} p<0.05$ and $* * p<0.01$.

tobacco plant. Auxin (IAA) plays an important role in plant growth and development, such as plant cell elongation and division, the formation of the root hair, and the growth of the main roots and lateral roots. The primary auxin-responsive genes include three gene families: Aux/IAA (auxin/IAA), GH3 (Gretchen Hagen 3), and SAUR (Small auxin up RNA). Aux/IAA proteins possess a potent transcriptional co-repression activity and suppress the early induction of auxin-responsive genes. Topping reduces the synthesis of IAA in the aboveground part. This shifts the distribution of IAA in the tobacco plant, resulting in alterations in the expression of certain genes related to the auxin signaling pathway. In our previous studies, the expression of two auxin-related miRNAs, Nta-miRn49, and Nta-miR164a, was shown to be markedly repressed by topping (Guo et al., 2011). Axi 1, the target of nta-miRn49, plays a role in auxin activity (Richard et al., 1994). NAC1, the target of nta-miR164a, mediates auxin signaling to promote lateral root development (Xie et al., 2000). In the current study, the expression of IAA9 decreased after topping. These changes in expression of genes related to root development are helpful to our understanding of the secondary growth of roots after topping.

\section{Wounding Stimulus Is the Main Factor Promoting the Capacity of Nicotine Synthesis after Topping}

Unlike animals, plants are unable to move in order to avoid environmental stresses, so they have instead evolved a comprehensive set of defenses to allow them to adapt to their environment. Topping is a form of damage sustained at the aboveground part, which triggers the wounding stress response. Nta-miR166c targets LRR family resistance proteins ( $\mathrm{McHale}$ et al., 2006), and our previous work has demonstrated that nta-miR166c is significantly repressed by topping (Guo et al., 2011). The current study also found that several stress responsive proteins (LRR1, HSP70, and FPPS) were up-regulated after topping.

Plant jasmonates have important signaling roles in defense responses against abiotic and biotic stresses. Nicotine is a 

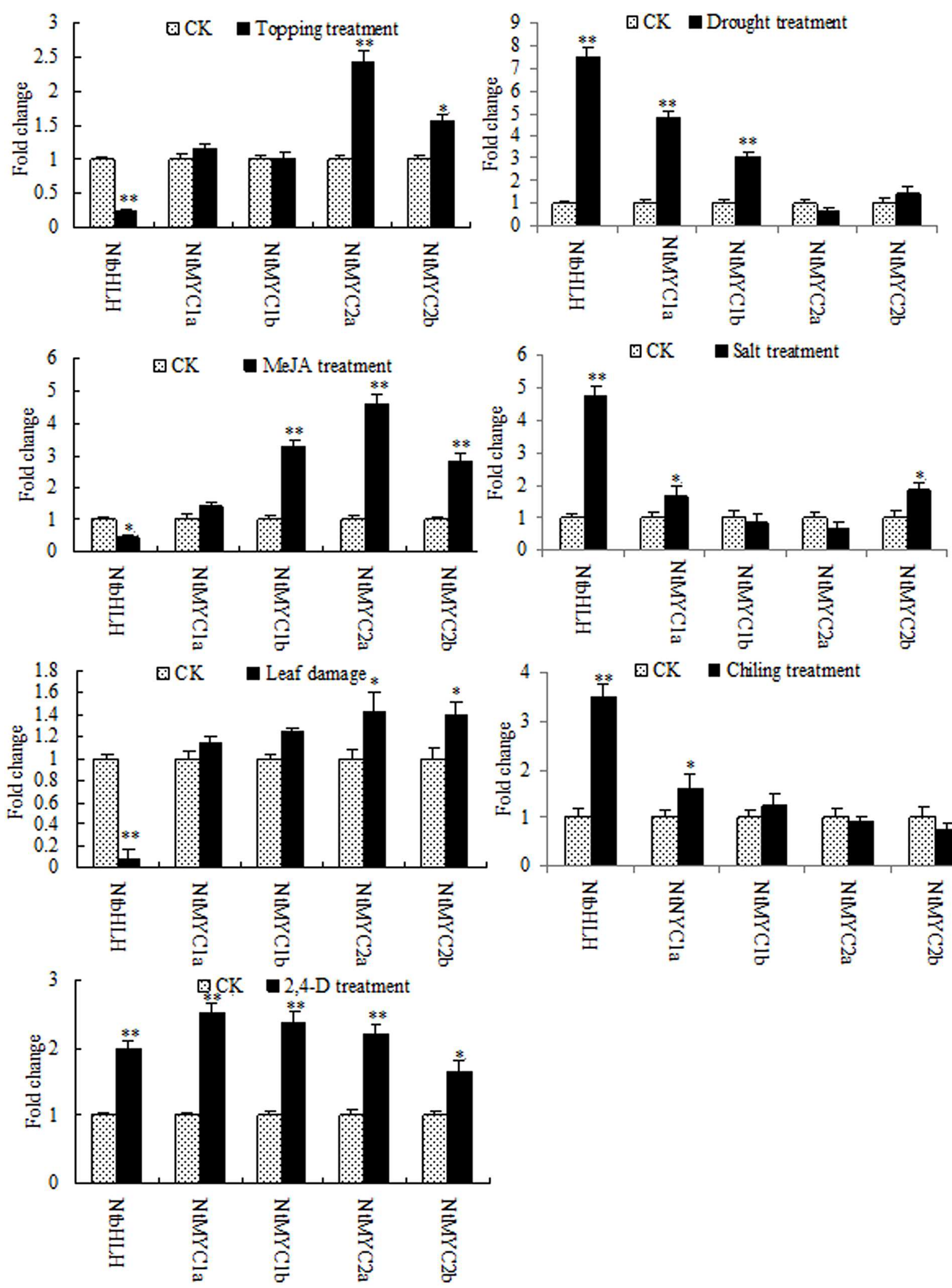

FIGURE 10 | RT-qPCR analyses of the five bHLH proteins in tobacco after stress treatment. Fold change was determined using the $2^{-\Delta} \Delta \mathrm{Ct}$ method and error bars represent the SD of the mean. Statistical significance: ${ }^{*} p<0.05$ and ${ }^{* *} p<0.01$. 
secondary metabolite produced by the defense responses. Coronatine-insensitive 1 (COI1), an F-box protein, is the key player in JA signaling. It has been demonstrated that the F-box protein is involved in jasmonate-inducible nicotine biosynthesis (Shoji and Hashimoto, 2011; Wang and Wu, 2013). COI1 is a part of an E3 ubiquitin ligase SCF ${ }^{\mathrm{COI} 1}$ complex, which is the receptor for the bioactive hormone JA-Ile. Most JA-induced responses are carried out by JA-Ile. JAZ proteins can repress JA signaling through interacting with MYC2, which is a positive transcription factor in JA-inducible nicotine biosynthesis. JA-Ile can promote the interaction between COI1 and JAZs, leading to degradation of JAZs and thus the activation of JA-inducible nicotine biosynthesis (Wang and $\mathrm{Wu}, 2013$ ). The F-box protein is the target protein of nta-miR2111, expression of which was shown to be significantly up-regulated by topping in previous studies (Guo et al., 2011). The results of root proteomics in the current study showed that F-box protein was differentially expressed before and after topping, suggesting that it is involved in post-topping enhancement of nicotine synthesis.

In Arabidopsis, the MYC2-family bHLH transcription factors are involved in the regulation of jasmonate-responsive genes. The four tobacco bHLH proteins (NtMYC1a/b and NtMYC2a/b) belong to the Arabidopsis MYC2 clade. NtMYC1a/b and NtMYC2a/b are grouped together with NbbHLH1 and NbbHLH2, respectively, (Shoji and Hashimoto, 2011). NbbHLH1 and NbbHLH2 in Nicotiana benthamiana are positive regulators in the jasmonate activation of nicotine biosynthesis (Todd et al., 2010). It was found that NtMYC2 recognizes the G-box sequences in the promoter regions of several nicotine biosynthesis genes, including PMT2 and QPT2 (Shoji and Hashimoto, 2011). In the present study, the expression of PMT, NtMYC1, and NtMYC2 are clearly regulated by biotic and abiotic stresses, although the responses of NtMYC1 and NtMYC2 show some differences. As shown in Figure 10, NtMYC2 was up-regulated more than NtMYC1 after topping, whereas NtMYC1 was up-regulated more than NtMYC2 after drought treatment, suggesting that

\section{REFERENCES}

Baldwin, I. T., Schmelz, E. A., and Ohnmeiss, T. E. (1994). Wound-induced changes in root and shoot jasmonic acid pools correlated with induced nicotine synthesis in Nicotiana sylvestris Spegazzini and Comes. J. Chem. Ecol. 20, 2139-2157. doi: 10.1007/BF02066250

Baldwin, I. T., Zhang, Z. P., Diab, N., Ohnmeiss, T. E., McCloud, E. S., Lynds, G. Y., et al. (1997). Quantification, correlations and manipulations of woundinduced changes in jasmonic acid and nicotine in Nicotiana sylvestris. Planta 201, 397-404. doi: 10.1007/s004250050082

Fukaki, H., Tameda, S., Masuda, H., and Tasaka, M. (2002). Lateral root formation is blocked by a gain-of-function mutation in the SOLITARY-ROOT/IAA14 gene of Arabidopsis. Plant J. 29, 153-168. doi: 10.1046/j.0960-7412.2001.01201.x

Guo, H. X., Kan, Y. C., and Liu, W. Q. (2011). Differential expression of miRNAs in response to topping in flue-cured tobacco (Nicotiana tabacum) roots. PLoS ONE 6:e28565. doi: 10.1371/journal.pone.0028565

Guo, H. X., Wang, S. X., Li, F. X., Li, S. M., Xiao, W. F., and Liu, W. Q. (2015). Enhanced protein extraction from tobacco roots for proteomic analysis. Anal. Lett. 48, 1-9. doi: 10.1080/00032719.2014.930871

Imanishi, S., Hashizume, K., Nakakita, M., Kojima, H., Matsubayashi, Y., and Hashimoto, T. (1998). Differential induction by methyl jasmonate of genes encoding ornithine decarboxylase and other enzymes involved in nicotine
NtMYC2 is the main transcription factor involved in JA-mediated nicotine synthesis after topping. In addition to the four bHLH proteins, the results of proteomic analysis showed that NtbHLH (nta000375) protein was down-regulated after topping. NtbHLH does not belong to MYC subfamily, and it may be a negative regulator in the JA-mediating activation of nicotine synthesis.

\section{CONCLUSION}

Topping of a tobacco plant triggers a comprehensive array of biological response processes, involving the transformation of the growth center and activation of the wounding stimulus response. IAA and JA signaling pathways play an important role in these responses.

\section{AUTHOR CONTRIBUTIONS}

The research was designed by WL. The experiments were performed by FL, HZ, SW, WX, and CD, and the data were analyzed by HG. The manuscript was written by HG.

\section{ACKNOWLEDGMENT}

This work was financially supported by the science and technology project of Tobacco Company of Henan Province (HYKJ201308) and Open Science and Technology Cooperation Projects of Henan Province (142106000187).

\section{SUPPLEMENTARY MATERIAL}

The Supplementary Material for this article can be found online at: http://journal.frontiersin.org/article/10.3389/fpls.2016.00582

biosynthesis in flue-cured tobacco cell cultures. Plant Mol. Biol. 38, 1101-1111. doi: 10.1023/A:1006058700949

Jin, Z. L., Hong, J. K., Yang, K. A., Koo, J. C., Choi, Y. J., Chung, W. S., et al. (2005). Over-expression of Chinese cabbage calreticulin 1, BrCRT1, enhances shoot and root regeneration, but retards plant growth in transgenic tobacco. Transgenic Res. 14, 619-626. doi: 10.1007/s11248-005-5694-6

Kim, J. H., Nguyen, N. H., Nguyen, N. T., Hong, S. W., and Lee, H. (2013). Loss of all three calreticulins, CRT1, CRT2 and CRT3, causes enhanced sensitivity to water stress in Arabidopsis. Plant Cell Rep. 32, 1843-1853. doi: 10.1007/s00299013-1497-z

Li, C. J., Teng, W., Shi, Q. M., and Zhang, F. S. (2007). Multiple signals regulate nicotine synthesis in tobacco plant. Plant Signal. Behav. 2, 280-281. doi: 10.4161/psb.2.4.4008

McHale, L., Tan, X. P., Koehl, P., and Michelmore, R. W. (2006). Plant NBS-LRR proteins:adaptable guards. Genome Biol. 7:212. doi: 10.1186/gb-2006-7-4-212

Qi, Y. C., Guo, H. X., Li, K., and Liu, W. Q. (2012). Comprehensive analysis of differential genes and miRNA profiles for discovery of topping-responsive genes in flue-cured tobacco roots. FEBS J. 279, 1054-1070. doi: 10.1111/j.17424658.2012.08497.x

Reilly, J. F., Martinez, S. D., Mickey, G., and Maher, P. A. (2002). A novel role for farnesyl pyrophosphate synthase in fibroblast growth factor-mediated signal transduction. Biochem. J. 366, 501-510. doi: 10.1042/bj20020560 
Richard, W., Hiroaki, H., Helge, L., Inge, C., and Jeff, S. (1994). Auxin inducibility and developmental expression of axi 1: a gene directing auxin independent growth in tobacco protoplasts. EMBO J. 13, 4729-4736.

Saunders, J. W., and Bush, L. P. (1979). Nicotine biosynthetic enzyme activities in Nicotiana tabacum L. genotypes with different alkaloid levels. Plant Physiol. 64, 236-240. doi: 10.1104/pp.64.2.236

Shi, Q. M., Li, C. J., and Zhang, F. S. (2006). Nicotine synthesis in Nicotiana tabacum L. induced by mechanical wounding is regulated by auxin. J. Exp. Bot. 57, 2899-2907. doi: 10.1093/jxb/erl051

Shoji, T., and Hashimoto, T. (2011). Tobacco MYC2 regulates jasmonate-inducible nicotine biosynthesis genes directly and by way of the NIC2-Locus ERF genes. Plant Cell Physiol. 52, 1117-1130. doi: 10.1093/pcp/pcr063

Shoji, T., Nakajima, K., and Hashimoto, T. (2000). Jasmonate induction of putrescine N-methyltransferase genes in the root of Nicotiana sylvestris. Plant Cell Physiol. 41, 831-839. doi: 10.1093/pcp/pcd001

Tatematsu, K., Kumagai, S., Muto, H., Sato, A., Watahiki, M. K., Harper, R. M., et al. (2004). MASSUGU2 encodes Aux/IAA19, an auxin-regulated protein that functions together with the transcriptional activator NPH4/ARF7 to regulate differential growth responses of hypocotyl and formation of lateral roots in Arabidopsis thaliana. Plant Cell 16, 379-393. doi: 10.1105/tpc.018630

Todd, A. T., Liu, E. W., Polvi, S. L., Pammett, R. T., and Page, J. E. (2010). A functional genomics screen identifies diverse transcription factors that regulate alkaloid biosynthesis in Nicotiana benthamiana. Plant J. 62, 589-600. doi: 10.1111/j.1365-313X.2010.04186.x

Uehara, T., Okushima, Y., Mimura, T., Tasaka, M., and Fukaki, H. (2008). Domain II mutations in CRANE/IAA18 suppress lateral root formation and affect shoot development in Arabidopsis thaliana. Plant Cell Physiol. 49, 1025-1038. doi: $10.1093 / \mathrm{pcp} / \mathrm{pcn} 079$

Wang, J. M., Sheehan, M., Brookman, H., and Timko, M. P. (2000). Characterization of cDNAs differentially expressed in roots of tobacco (Nicotiana tabacum cv Burley 21) during the early stages of alkaloid biosynthesis. Plant Sci. 158, 19-32. doi: 10.1016/S0168-9452(00)00293-4
Wang, L., and Wu, J. Q. (2013). The essential role of jasmonic acid in plantherbivore interactions-using the wild tobacco Nicotiana attenuata as a Model. J. Genet. Genomics 40, 597-606. doi: 10.1016/j.jgg.2013.10.001

Winz, R. A., and Baldwin, I. T. (2001). Molecular interactions between the specialist herbivore Manduca sexta (Lepidop-tera, Sphingidae) and its natural host Nicotiana attenuata. IV. Insect-induced ethylene reduces jasmonateinduced nicotine accumulation by regulating putrescine $\mathrm{N}$-methyltrans-ferase transcripts. Plant Physiol. 125, 2189-2202.

Xie, Q., Frugis, G., Colgan, D., and Chua, N. H. (2000). Arabidopsis NAC1 transduces auxin signal downstream of TIR1 to promote lateral root development. Genes Dev. 14, 3024-3036. doi: 10.1101/gad.852200

Xu, B., and Timko, M. (2004). Methyl jasmonate induced expression of the tobacco putrescine N-methyltransferase genes requires both G-box and GCC-motif elements. Plant Mol. Biol. 55, 743-761. doi: 10.1007/s11103-004-1962-8

Zhang, D., Liu, G. S., Zhang, J. X., and Xu, M. (2006). Effect of different topping time on activity of root system and accumulation of nicotine in tobacco plants. Chin. Tob. Sci. 27, 38-41.

Zhang, Z. P., and Baldwin, I. T. (1997). Transport of [2-14C] jasmonic acid from leaves to roots mimics wound induced changes in endogenous jasmonic acid pools in Nicotiana sylvestris. Planta 203, 436-441. doi: 10.1007/s004250 050211

Conflict of Interest Statement: The authors declare that the research was conducted in the absence of any commercial or financial relationships that could be construed as a potential conflict of interest.

Copyright (c) 2016 Li, Zhang, Wang, Xiao, Ding, Liu and Guo. This is an open-access article distributed under the terms of the Creative Commons Attribution License (CC BY). The use, distribution or reproduction in other forums is permitted, provided the original author(s) or licensor are credited and that the original publication in this journal is cited, in accordance with accepted academic practice. No use, distribution or reproduction is permitted which does not comply with these terms. 